\title{
Heart Transplant: Protocol for Monitoring Posttransplant Complications
}

\author{
Adriana Habor ${ }^{1 *}$, Anca $\operatorname{Sin}^{2}$ and Horatiu Suciu ${ }^{3}$ \\ ${ }^{1}$ Department of Physiology, University of Medicine and Pharmacy, Targu Mures, Romania \\ ${ }^{2}$ Department of Cell and Molecular Biology, University of Medicine and Pharmacy, Targu Mures, Romania \\ ${ }^{3}$ Surgery Clinic Department, University of Medicine and Pharmacy, Targu Mures, Romania
}

*Corresponding author: Adriana Habor, Department of Physiology, University of Medicine and Pharmacy, Targu Mures, Romania.

Received Date: October 24, 2019

Published Date: October 30, 2019

\begin{abstract}
Endomyocardial biopsy, first introduced in the posttransplant monitoring protocol by Billingham from Standford University, has become a necessity in the histological diagnosis, especially after the introduction of immunosuppressive treatment. Rejection is histologically illustrated by the occurrence of an interstitial and/or perivascular lymphocytic inflammatory infiltrate which, in severe cases, is associated with significant myocyte damage. In certain situations, the acute rejection is followed by irreversible damage, leading to patient's death. The histological monitoring protocol was applied in 63 patients with heart transplant performed at the Emergency Institute for Cardiovascular Diseases and Transplant of Targu Mures, between 1999 and the present time. Six of these interventions were performed in 2016. Twenty-two of the 63 patients deceased during follow-up due to various complications, whereas 32 were diagnosed with cytomegalovirus infection, based on clinical and histological findings. All patients underwent biopsy at various intervals of time, either following a standardized protocol or driven by the lesion pattern revealed by the pathological report. Three to six tissue samples were fixed in $10 \%$ buffered formaldehyde prior to paraffin embedding. Paraffin blocks were sectioned at three or four levels. Hematoxyllin-eosin, trichrome Masson and methyl-pyronin green staining were used.

Conclusion: Endomyocardial biopsy monitoring of heart transplant recipients is still the most important tool for the complex diagnosis of post-transplant complications, facilitating the adoption of timely and targeted therapeutic intervention, and the permanent improvement of immunosuppressive therapeutic protocols, with the goal of increasing survival time.
\end{abstract}

Keywords: Cardiac transplantation, Acute rejection, Chronic rejection, Intramyocardial biopsy

\section{Introduction}

In many countries, heart transplantation is the therapeutic method of choice for irreducible heart failure of various causes, among which the most common are dilating cardiomyopathy and coronary heart disease. Endomyocardial biopsy, first introduced in the posttransplant monitoring protocol by Billingham et al. from Standford University, has become a necessity in the histopathological diagnosis, especially after the introduction of immunosuppressive treatment [1]. Most of the times, endomyocardial biopsy remains the only accurate diagnostic method prior to development of significant myocardial injury. Once detected, reversible rejection reaction can be corrected through targeted therapy, by suitably increasing immunosuppression [2]. Despite modern immunosuppressive regimes, acute rejection remains a leading cause of morbidity and mortality in heart transplant recipients [3]. Rejection is histopathologically illustrated by the occurrence of an interstitial and/or perivascular lymphocytic inflammatory infiltrate which, in severe cases, is associated with significant myocyte damage. In certain situations, the acute rejection is followed by irreversible damage, leading to patient's death [4]. The main aim of this paper was to highlight the major role of biopsy in the short-, medium- and long-term follow-up of the heart transplant recipients, and in the diagnosis of complications which might occur in various moments after the transplant.

\section{Materials and Methods}

The histological monitoring protocol was applied in 63 patients with heart transplant performed at the Emergency Institute for Cardiovascular Diseases and Transplant of Tirgu Mures, between 1999 and the present time. Twenty-two of the 63 patients deceased during follow-up due to various complications, whereas 32 were diagnosed with cytomegalovirus infection $[5,6]$, based on clinical 
and histological findings. All patients underwent biopsy at various intervals of time, either following a standardized protocol or driven by the lesion pattern revealed by the pathological report. Three to six tissue samples were fixed in $10 \%$ buffered formaldehyde prior to paraffin embedding. Paraffin blocks were sectioned at three or four levels. Hematoxyllin-eosin, trichrome Masson and methyl-pyronin green staining were used. In spite of all difficulties related to monitoring and postoperative treatment, prognosis is encouraging. Biopsies were taken from the interventricular septum or the right ventricle wall. According to the time of follow-up, lesions were monitored during the early (the first month), medium (the first year), and late period (after the first year). Overall survival is $80 \%$ over one year and more than $60 \%$ at 5 years [7] and $50 \%$ at 10 years [8], and $22.4 \%$ at 20 years [9]. Care was taken in the assessment of possible artifacts, such as myocardial contraction bands, which are difficult to distinguish from ischemiareperfusion lesions, and the interstitial edema due to tissue stain upon sampling. We also considered previously sampled areas, with necrotic adjacent myocytes, wherein non-specific granulation tissue develops, eventually turning into hyaline scar. In some of these scars, fibrous extensions of various thicknesses intertwining with myocardial fibers and macrophages or foreign-body giant cells can be observed. Myocardial perforation is emphasized by the presence of mesothelial cells and adipocytes. This finding should not be a source of alert, since many areas of adhesion occur in the postoperative pericardial sac. In the first 10 days posttransplant, all histology changes that could reveal an immediate graft failure, a hyperacute rejection, acute cellular or humoral rejection, or ischemia and reperfusion changes were taken into account. Histological monitoring materialized by quantifying tissue pathological changes on all biopsies at different time intervals according to a well-established protocol for endomyocardial biopsy. All changes in myocardial fibers were assessed, interstitial and perivascular fibrosis was quantified and reported, development of hemorrhagic foci, edema, and presence of adipocytes were evaluated. The Quality effect was diagnosed in a few cases. Once diagnosed, allograft rejection was histologically staged according to ISHLT (International Society of Heart and Lung Transplantation) standards [10]. Any suspicion of rejection imposed a new biopsy.

\section{Result}

Successive biopsies enabled the monitoring of treatment response. Besides mild, moderate or severe acute rejection, other histopathological forms such as continuous acute rejection, remission of acute rejection, and resolved acute rejection were diagnosed. Each time, lesions of the endomyocardial blood vessels were assessed: endothelial thickening, degenerative changes, the onset of lymphocytic perivascular infiltrate, vascular necrosis, the occurrence of thrombotic endovasculopathy in serious cases, intimal concentric fibrous thickening of various degrees, intramural lipid deposits with foamy subendothelial histiocytes, exocytosis, and endothelial wall edema as sign of humoral rejection. On each biopsy, possible injuries similar to, but unrelated to rejection, such as those due to ischemia and reperfusion, calcifications, endocardial infiltrate related to cyclosporin therapy, and myocyte hypertrophy, were described. Early detection of accelerated atherosclerosis as a sign of chronic rejection, as well as adapting the immunosuppressive treatment to the histological changes, is of vital importance. As graft rejection is a main cause of morbidity in heart transplant patients, therapy must be carefully conducted, based on the morphological changes detected by biopsy, immunohistochemistry and modern molecular methods [11]. Cytomegalovirus, present in most transplant patients either as a first infection or reactivation of an infection transmitted through the graft itself or the blood used during surgery, can also be indirectly detected by histological assessment of biopsy samples, in the presence of focal necrosis, micro-abcesses and characteristic "owl-eye" cells. The importance of diagnosing this infection relies on its possible role in the induction of obliterating arteriopathy and, subsequently, chronic graft rejection [12].

Hyperacute rejection may occur shortly after transplantation, due to a pre-sensitization against donor HLA antigens, or to ABO blood group incompatibility [13] rejection which is seen also in kidney transplant recipients [14]. The humoral mechanism is mainly directed against the vascular endothelium, leading to massive necrosis of the transplanted organ. In this case, histological examination, which is very important for early and accurate diagnosis, can reveal strong interstitial hemorrhage and edema, with no inflammatory reaction. Acute humoral rejection, which is less common, occurs in about $7 \%$ of transplant patients, especially in those with a positive "cross-match" test, in those with cytomegalovirus infection, or with immune hyperactivity. The diagnosis is more difficult to establish, and can rely on endomyocardial biopsy, wherein vessel wall edema, swelling of endothelial cells in the presence or absence of plasma cells infiltrate, can be observed. The late detection of a humoral rejection entrails the risk of chronic rejection installation [15].

Regarding chronic rejection, the early detection of fibrotic, obliterating lesions, foamy histiocytes, cytomegalovirus infection, in correlation with clinical evolution, may prompt for proper therapy in order to save the affected organ and the patient $[16,17]$.

\section{Discussion}

Despite the development of a variety of non-invasive methods for the detection of rejection, the endomyocardial biopsy will remain important in the management of patients following cardiac transplantation, as non-invasive techniques are associated with low specificity for the diagnosis of rejection [18-22]. Some biomarkers associated with the early immune response to cardiac allograft retain the power to be diagnostic and, even better, predictive of acute rejection, as in the case of pretransplant CXCL10 serum level, but still multicenter studies are needed for validation and standardization of such biomarkers in daily clinical practice [23]. A new standardized classification will likely improve the utility of the biopsy by simplifying interpretation of cellular rejection and importantly allowing recognition of antibody-mediated rejection [24]. 


\section{Conclusion}

Endomyocardial biopsy monitoring of patients with heart transplant remains the most important tool for the complex diagnosis of post-transplant complications, facilitating the adoption of timely and targeted therapeutic intervention, and the permanent improvement of immunosuppressive therapeutic protocols, with the goal of increasing survival time.

\section{Acknowledgements}

This paper was supported by a research grant funded by University of Medicine and Pharmacy, Targu-Mures, Romania, "Monitorizarea reacţiei de respingere în transplantul cardiac", Contract number: 6574/2015.

\section{Conflict of Interest}

No conflict of interest.

\section{References}

1. Billingham ME (1990) Endomyocardial biopsy diagnosis of acute rejection in cardiac allografts. Prog Cardiovasc Dis 33(1): 11-18.

2. Tazelaar HD (1990) Spectrum and diagnosis of myocardial rejection. Cardiol Clin 8(1): 119-139.

3. Miller CA, Fildes JE, Ray SG, Doran H, Yonan N, et al. (2013) Non-invasive approaches for the diagnosis of acute cardiac allograft rejection. Heart Br Card Soc 99(7): 445-453.

4. Winters GL (1997) The challenge of endomyocardial biopsy interpretation in assessing cardiac allograft rejection. Curr Opin Cardiol 12(2): 146-152.

5. Delgado JF, Reyne AG, de Dios S, López-Medrano F, Jurado A, et al. (2015) Influence of cytomegalovirus infection in the development of cardiac allograft vasculopathy after heart transplantation. J Heart Lung Transplant Off Publ Int Soc Heart Transplant 34(8): 1112-1119.

6. Yoosabai A, Mehta A, Kang W, Chaiwatcharayut W, Sampaio M, et al. (2015) Pretransplant malignancy as a risk factor for posttransplant malignancy after heart transplantation. Transplantation 99(2): 345-350.

7. Ghadimi H, Tavangar SM (2005) Histopathological findings in cardiac transplant recipients: an assessment after 10 years' experience in Iran. Transplant Proc 37(10): 4535-4536.

8. Logeais Y, Lelong B, Langanay T, Corbineau H, Rioux C, et al. (2003) Results of heart transplantation: experience of 233 grafts. Bull Académie Natl Médecine 187(2): 325-340.

9. Hetzer R, Delmo Walter EM (2013) Trends and outcomes in heart transplantation: the Berlin experience. HSR Proc Intensive Care Cardiovasc Anesth 5(2): 76-80.

10. Mehra MR, Crespo-Leiro MG, Dipchand A, Ensminger SM, Hiemann NE et al. (2010) International Society for Heart and Lung Transplantation working formulation of a standardized nomenclature for cardiac allograft vasculopathy-2010. J Heart Lung Transplant Off Publ Int Soc Heart Transplant 29(7): 717-727.

11. Revelo MP, Stehlik J, Miller D, Snow GL, Everitt MD, et al. (2011) Antibody testing for cardiac antibody-mediated rejection: which panel correlates best with cardiovascular death. J Heart Lung Transplant Off Publ Int Soc Heart Transplant 30(2): 144-150.

12. Johansson I, Andersson R, Friman V, Selimovic N, Hanzen L, et al. (2015) Cytomegalovirus infection and disease reduce 10-year cardiac allograft vasculopathy-free survival in heart transplant recipients. BMC Infect Dis 15: 582 .

13. Hageman M, Michaud N, Chinnappan I, Klein T, Mettler B, et al. (2015) ABO-incompatible heart transplants. Perfusion 30(3): 209-212.

14. Opelz G, Morath C, Süsal C, Tran TH, Zeier M, et al. (2015) Three-year outcomes following 1420 ABO-incompatible living-donor kidney transplants performed after ABO antibody reduction: results from 101 centers. Transplantation 99(2): 400-404.

15. Szymańska S, Grajkowska W, Pronicki M (2014) Pathologic diagnosis of antibody-mediated rejection in endomyocardial biopsy after heart transplantation based on renewed International Society for Heart and Lung Transplantation criteria. Pol J Pathol Off J Pol Soc Pathol 65(3): 176-181.

16. Agozzino L, Thomopoulos K, Esposito S, Agozzino M, De Vivo F, et al. (1999) Pathology of heart transplantation. (Morphological study of 1246 endomyocardial biopsies from 167 transplanted hearts). Causes of early, intermediate, and late deaths. Pathologica 91(2): 89-100.

17. Cotoi OS, Suciu H, Sin A, Turcu M, Morariu SH, et al. (2011) Cardiac allograft vasculopathy in adult human recipients: a case series of seven patients and review of literature. Romanian J Morphol Embryol Rev Roum Morphol Embryol 52(2): 685-690.

18. Balduini A, Campana C, Ceresa M, Arbustini E, Bosoni T, et al. (2003) Utility of biochemical markers in the follow-up of heart transplant recipients. Transplant Proc 35(8): 3075-3078.

19. Dengler TJ, Gleissner CA, Klingenberg R, Sack F-U, Schnabel PA, et al. (2007) Biomarkers after heart transplantation: nongenomic. Heart Fail Clin 3(1): 69-81.

20. Crespo-Leiro MG, Stypmann J, Schulz U, Zuckermann A, Mohacsi P, et al. (2016) Clinical usefulness of gene-expression profile to rule out acute rejection after heart transplantation: CARGO II. Eur Heart J 37(33): 2591-2601.

21. Kennel PJ, Schulze PC (2015) Novel Biomarker Approaches for Managing Patients With Cardiac Transplantation. Curr Heart Fail Rep 12(5): 328332.

22.Lu W, Zheng J, Pan X, Sun L (2015) Diagnostic performance of echocardiography for the detection of acute cardiac allograft rejection: a systematic review and meta-analysis. PloS One 10(3): e0121228.

23. Crescioli C (2013) The role of immunological biomarkers in cardiac rejection. Curr Opin Organ Transplant 18(5): 595-600.

24. Patel JK, Kobashigawa JA (2006) Should we be doing routine biopsy after heart transplantation in a new era of anti-rejection. Curr Opin Cardiol 21(2): 127-131. 